\title{
Factors Associated with Morbidity in ICU
}

\section{AK Mohiuddin*}

Secretary and Treasurer, Dr. M. Nasirullah Memorial Trust, Tejgaon, Dhaka, Bangladesh

*Corresponding Author: AK Mohiuddin, Secretary and Treasurer, Dr. M. Nasirullah Memorial Trust, Tejgaon, Dhaka, Bangladesh.

Received: September 16, 2019; Published: September 23, 2019

DOI: 10.31080/ASMS.2019.03.0414

The beginnings of caring about critically sick patients go back to Florence Nightingale's work during the Crimean War in 1854, yet the subspecialty of basic care drug is moderately young. Numerous elements are theorized to add to the generally high occurrence and related morbidity of medication errors in the ICU. The patients themselves are the most complex and critically sick in the hospital setting [1]. Basic care regions present a specific test with respect to medication errors. They are a powerful domain with critically sick patients who frequently require quick adaptation of progressing the executives. ICUs can be blunder inclined settings, where even generally minor antagonistic occasions can prompt genuine handicap. By righteousness of being more wiped out, more seasoned, and having more comorbidities, these patients are less versatile to errors. Since they require a higher power of care arrangement and may get more medications, they might be at more serious danger of iatrogenic mischief. Pharmacokinetics of medications can likewise be modified in critically sick patients, basically through changes in volume of distribution and medication leeway. Huge volume revivals, positive weight ventilation, surgeries, foundational provocative reaction, and changes in protein official, all basic in ICU patients, influence the pharmacokinetics of numerous drugs. Also, these patients are generally unfit to help facilitate their very own care, an issue exasperated by the volume of exchanges to and from ICUs. Medication wellbeing in ICUs may likewise be undermined due to the risks related with the utilization of numerous medications per quiet and the utilization of highhazard drugs related with possibly serious antagonistic occasions [2]. Drugs utilized in the ICU are bound to be powerful, require portion counts, have medication interactions, and be consistent imbuements (which have a more prominent potential for blunder). Numerous medications might be utilized for off-label indications in the ICU setting, like the non-ICU inpatient and outpatient settings. The mix of these components makes patients in basic care zones especially defenseless against medication errors and their conceivably dire consequences. Patients admitted to ICU with chronic obstructive pulmonary disease (COPD) frequently have numerous comorbidities and present with intense respiratory disappointment because of an infective exacerbation or toward the end phase of their disease [3]. Atrial fibrillation (AF) is the most common arrhythmia encountered in the ICU. Preexisting AF is highly prevalent among older patients with chronic conditions who are at risk for critical illness, whereas new-onset AF can be triggered by accelerated atrial remodeling and arrhythmogenic triggers encountered during critical illness. The acute loss of atrial systole and onset of rapid ventricular rates that characterize new-onset $\mathrm{AF}$ often lead to decreased cardiac output and hemodynamic compromise. Thus, new-onset AF is both a marker of disease severity as well as a likely contributor to poor outcomes, similar to other manifestations of organ dysfunction during critical illness [4]. Evaluating immediate hemodynamic effects of new-onset AF during critical illness is an important component of rapid clinical assessment aimed at identifying patients in need of urgent direct current cardioversion, treatment of reversible inciting factors, and identification of patients who may benefit from pharmacologic rate or rhythm control. In addition to acute hemodynamic effects, new-onset AF during critical illness is associated with both short- and long-term increases in the risk of stroke, heart failure, and death, with $\mathrm{AF}$ recurrence rates of approximately $50 \%$ within 1 year following hospital discharge. In general ICU patients, incidence of new-onset AF was more than 11\% with a high impact on morbidity and mortality, particularly associated with the presence of ARF. Variables that have been commonly linked to an increased risk for in-hospital mortality in mechanically 
ventilated patients include age, comorbidities, SAPS (Simplified Acute Physiology Score) III, severe adult respiratory distress syndrome (ARDS), deep sedation, duration of mechanical ventilation (MV) and ICU complications [5]. However, there is a wide variation in the prognostic variables between studies, which may be related to differences in the characteristics of patient cohorts, clinical variables recorded, and the geographical setting of different studies. Patients in ICU are usually at high risk of mortality not only from their critical illness but also from secondary complication such as nosocomial infection. Nosocomial pneumonia, a common ICU infection, affects around $40 \%$ of all critically ill patients, where more than $85 \%$ of it is associated with mechanical ventilation. The mortality rate for VAP (hospital-acquired/nosocomial pneumonia) develops more than $48-72 \mathrm{~h}$ after endotracheal intubation) ranges between $27 \%$ to $76 \%$. Pseudomonas or Acinetobacter pneumonia is associated with higher mortality rates than those associated with other organisms [6]. Delirium is a multifactorial substance, and its understanding keeps on advancing. Delirium has been related with expanded morbidity, mortality, length of stay, and cost for hospitalized patients, particularly for patients in the ICU. $25 \%$ to $89 \%$ ICU patients are accounted for to be influenced by delirium [7]. While medication management obviously assumes a job in delirium management, there are an assortment of nonpharmacologic mediations, pharmacologic minimization techniques, and conventions that have been as of late depicted. There has been an expansion in the quantity of patients experiencing open heart activity with the prolongation of future and medical advances. It has been accounted for that around $19 \%$ - $45 \%$ of the cases may experience delayed serious care after open heart activity [8]. In certain investigations, advanced age, female gender, decreased left ventricular function, arrhythmia, inotropic agent support and intra-aortic inflatable pump necessities have been recognized as hazard factors for delayed serious care. Heart failure following neurosurgery (craniotomy and spine surgery) is a staggering intricacy related with critical postoperative morbidity and mortality. Critically sick children with weight have higher danger of mortality and length of hospital stay contrasted with children without corpulence. In critically sick patients anticipating move from the ED to the medical ICU, electronic ICU care was related with diminished mortality and lower ICU resource utilization. From a clinical point of view, dysphagia is notable to be related with an expanded danger of aspiration and aspiration-induced pneumonia, delayed resumption of oral admission/ailing health, diminished QoL, delayed ICU and hospital length of stay, and expanded morbidity and mortality [9]. Grown-up medical ICU patients with upgrades in every day protein admission during hospitalization who endure hospitalization have diminished chances of mortality in the 3 months following hospital release. Diabetes mellitus may have antagonistic impact on ICU patients causing organ failure and inconveniences [10]. Be that as it may, how DM naturally influences the ICU mortality, is as yet open for discussion.

\section{Bibliography}

1. Kruer RM., et al. "Reducing medication errors in critical care: a multimodal approach". Journal of Clinical Pharmacology 6 (2014): 117-126.

2. Alvim MM., et al. "Adverse events caused by potential drugdrug interactions in an intensive care unit of a teaching hospital". The Revista Brasileira de Terapia Intensiva 27.4 (2015): 353-359.

3. Brown H., et al. "Factors associated with hospital mortality in critically ill patients with exacerbation of COPD". International Journal of Chronic Obstructive Pulmonary Disease 13 (2018): 2361-2366.

4. Bosch NA., et al. "Atrial Fibrillation in the ICU". Chest 154.6 (2018): 1424-1434.

5. Hammad E., et al. "Risk factors for hospital mortality among mechanically ventilated patients in respiratory ICU". Pulmonary Critical Care 9.3 (2015): 231-237.

6. Mohiuddin AK. "Pharmacists in Critical Care". Innovations in Pharmacy 10.1 (2019): 1-15.

7. Barbateskovic M., et al. "Pharmacological interventions for prevention and management of delirium in intensive care patients: a systematic overview of reviews and meta-analyses". BMJ Open 9.2 (2019): e024562.

8. Tunç M., et al. "Risk Factors for Prolonged Intensive Care Unit Stay After Open Heart Surgery in Adults". Turkish Journal of the Anaesthesiology and Reanimation 46.4 (2018):283-291.

9. Zuercher P., et al. "Dysphagia in the intensive care unit: epidemiology, mechanisms, and clinical management". Critical Care 23.1 (2019): 103. 
10. Dutt T., et al. "Do we need to screen every patient in intensive care unit for diabetes in community with high prevalence of diabetes?" World Journal of Diabetes 10.3 (2019):137-139.

Volume 3 Issue 10 October 2019

(C) All rights are reserved by AK Mohiuddin. 